\title{
Histological alterations to the hamstring tendon caused by cleaning during autograft preparation
}

\author{
Y. Okazaki, T. Furumatsu, A. Maehara, S. Miyazawa, Y. Kamatsuki, T. Hino, T. Ozaki \\ Department of Orthopaedic Surgery, Okayama University Graduate School, Okayama, Japan.
}

\author{
CORRESPONDING AUTHOR: \\ Takayuki Furumatsu \\ Department of Orthopaedic Surgery, \\ Okayama University Graduate School, \\ Okayama, Japan \\ Phone: +81 862357273 \\ Fax: +81 862239727 \\ E-mail: matio@md.okayama-u.ac.jp
}

DOI:

10.32098/mltj.02.2019.10

LEVEL OF EVIDENCE:

$3 b$

\begin{abstract}
SUMMARY
Introduction. This study evaluates histological alterations in the hamstring tendon caused by cleaning the muscle fibers during preparation of an autograft for anterior cruciate ligament reconstruction. Methods. We collected semitendinosus tendons from 6 patients who underwent anterior cruciate ligament reconstruction and used a curette to scratch the tendon for cleaning. Three groups of increasing repetitions of scratching $(5,10$, and 15$)$ were compared to a control (no scratching). Histological alterations in the tendon were evaluated using the modified Bonar score. Immunohistochemical analysis was also performed for type I collagen, with measurement of the area stained for type I collagen and cell numbers in the collagen fibers. Results. The mean modified Bonar score was 0.8 in the control group, and 3.2, 5.7, and 7.2 for the 5-, 10-, and 15-repeated scratching groups, respectively. The area of type I collagen staining decreased with increasing number of scratches $(\mathrm{P}<0.05$ for all groups), with a decrease of $>50 \%$ in cellular density between the 5 - and 10-repetition groups $(\mathrm{P}=0.004)$. Conclusion. Cleaning the hamstring tendons by scratching caused histological alterations and damage to type I collagen. Specifically, cellular density decreased markedly on scratching 10 times, suggesting that excessive curettage, leading to $>10$ scratches, might be deleterious.
\end{abstract}

\section{KEY WORDS}

anterior cruciate ligament; bamstring tendon; knee joint; type I collagen; fibroblast

\section{INTRODUCTION}

The anterior cruciate ligament (ACL) is a knee joint ligament that is frequently damaged. ACL rupture causes instability of the knee, which increases the risk of meniscus injury and early-stage osteoarthritis (1). Conservative or surgical treatment is performed to restore knee stability to the preinjury level (2). ACL reconstruction is favored for younger and more active patients, allowing for an earlier return to activities and competitive sport (3). Some studies have reported comparable outcomes for double-bundle and single-bundle ACL reconstruction, with no differences in clinical knee scores and knee extension and flexion muscular strength between the two graft types $(4,5)$. Hamstring autograft is primarily used for double-bundle ACL recon- struction. In highly physically active patients, $\leq 45$ years of age, allografts are not recommended because of the high risk of re-rupture(6). Although the maximum failure strength of hamstring tendons is lower than that of bone-patellar tendon-bone grafts, multiple folding of the tendon increases the tensile strength of the graft sufficiently for ACL reconstruction and, therefore, hamstring tendons are frequently the first choice for ACL graft material (7).

Hamstring tendons are categorized as dense connective tissue. Histologically, they have smaller cell nuclei, less proteoglycan, and more type I collagen fibers than the ACL (8). However, after ACL reconstruction, a graft undergoes transformation, from a stage of initial necrosis to revascularization, cell growth, collagen reconstitution, and matura- 
tion, a process known as "ligamentization" (9). Through this graft remodeling process, which lasts for a year or more, a reconstructed graft transforms into tissue that resembles a healthy ACL but is not exactly the same (10).

Research into the healing process of human grafts has increased in recent years. Clinical factors known to affect graft healing include graft isometricity, anatomical positioning, patient compliance, the healing response, vascularity, biomechanical strength, and post-operative rehabilitation (11). However, much remains unclear about how the healing process is affected by damage during the preparation of the hamstring autograft itself. In practice, excess muscle fibers must be cleaned from the tendon using a periosteal elevator or curette during preparation (12). This cleaning involves scratching the muscle fibers on the tendon multiple times using one of these tools. Scratching can cause microscopic damage to the tendon, but the degree of histological alteration is unclear. Therefore, the purpose of this study was to assess the histological alteration caused by tendon cleaning during the preparation of hamstring autografts. Our hypothesis was that the more times a hamstring tendon is scratched during cleaning, the greater the histological damage. Furthermore, the specific aim of this study was to determine the number of scratches that cause deleterious damage to the hamstring autograft.

\section{MATERIALS AND METHODS}

\section{Graft preparations}

Institutional review board approval and informed consent were obtained before all experimental studies.

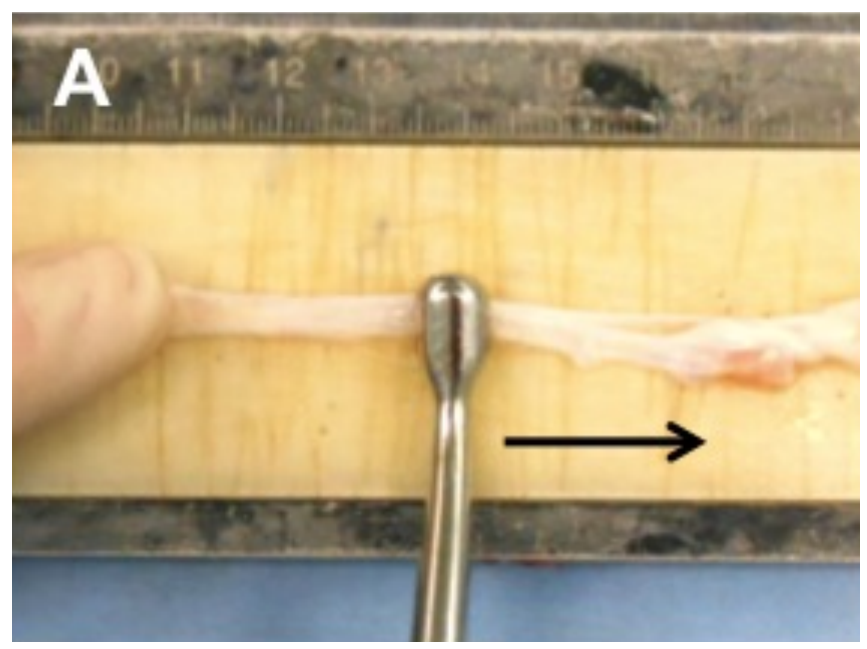

Six consecutive patients ( 2 women, 4 men; mean age, 22 years, range, 19 to 26 years), who underwent double-bundle ACL reconstruction using semitendinosus (ST) and gracilis tendon autografts, between February and April 2017, were enrolled into the study. For the ACL reconstructions, a 2-3 $\mathrm{cm}$ vertical skin incision was made above the pes anserinus, medial to the tibial tubercle, and both tendons were collected using a tendon stripper. The ACL reconstruction graft was created using both the ST and $G$ tendons. The average length of the ST tendon was $24 \mathrm{~cm}$ (range, 23-26 $\mathrm{cm})$. The ST tendon was doubled, and the length of the graft was made over $6.5 \mathrm{~cm}$, so that the ST tendon was left at over approximately $10 \mathrm{~cm}$. The histological analysis was conducted using the tendon part of the excised ST tendon. For cleaning, the extracted tendons were fixed to a preparation board (GRAFTMASTER; Smith \& Nephew, USA) and scratched in one direction (left to right) using a curette (ACUFEX NOTCHMASTER $8 \mathrm{~mm}$ Curette; Smith \& Nephew, USA), with an 8.0-mm wide tip (Figure 1A). Five surgeons performed a scratching test of 5, 10, and 15 repetitions along the ST tendon, with an average applied force of $2.5 \mathrm{~N}$ (range, 1.3-3.3 N), measured using a Newton meter. A non-scratched section was used as a control. The control and the three different areas of scratching $(5,10$, and 15 repetitions) were then cut every $1 \mathrm{~cm}$ for histological analysis (Figure 1B).

\section{Histological analysis}

The ST specimens were fixed with a $10 \%$ formalin solution and embedded in paraffin blocks. Sections were created by making 6- $\mu \mathrm{m}$ slices, parallel to the direction of the scratch-

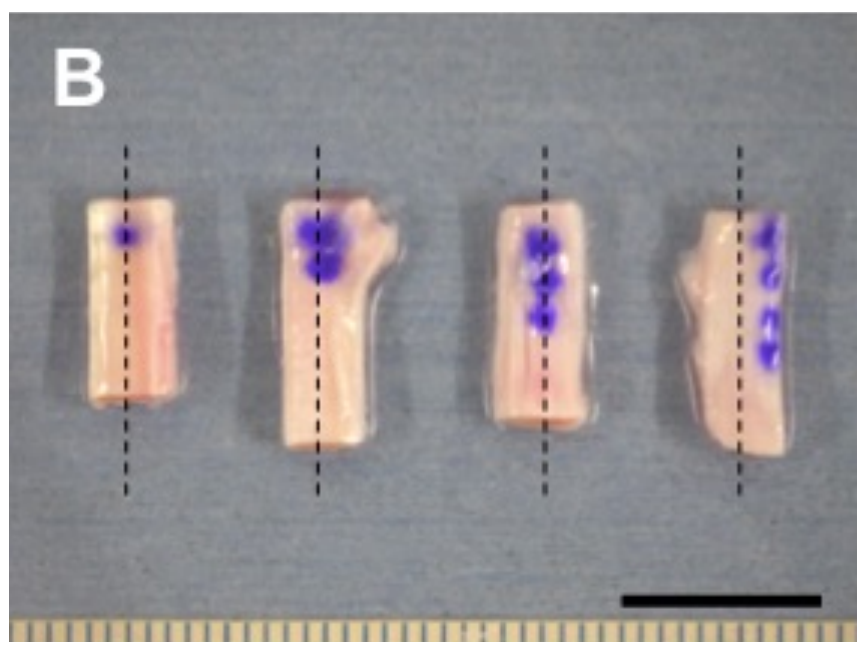

Figure 1. The scratching methods. (A) Semitendinosus (ST) tendons were scratched from left to right using a curette. (B) The cut pieces of ST tendon are shown from left to right, as follows: 0, 5, 10, and 15 times. Bar, $10 \mathrm{~mm}$. 
es. Sections were then subjected to hematoxylin-eosin staining. The degree of histological alteration was assessed using the modified Bonar scoring system (13). This score describes the extent of degeneration of the tendon based on the following 5 features: cell morphology, collagen arrangement, cellularity, vascularity, and ground substance. Each feature has a maximum score of 4 points, and the scores for each of the 5 features are summed to obtain the total score, with a higher score being indicative of greater damage. The Bonar score for the control and 3 scratch types $(5,10$, and 15 repetitions) was then compared.

\section{Immunohistochemical analysis}

Rabbit anti-type I collagen antibody (ab34710; Abcam, Cambridge, UK) was used to assess deposits of type I collagen in the areas on the tendon that were scratched, as previously described (14). Six- $\mu \mathrm{m}$ sections were deparaffinized in xylene and incubated with $20 \mu \mathrm{g} / \mathrm{ml}$ proteinase $\mathrm{K}$ (Abcam). For staining, the rabbit antibody was diluted in a blocking buffer and incubated overnight at $4^{\circ} \mathrm{C}$. Samples were washed $3 \times 5 \mathrm{~min}$ in PBS and incubated for $1 \mathrm{~h}$ with secondary antibodies in blocking buffer. Slides were then washed three times in PBS, counterstained with hematoxylin. Photoshop CC (Adobe, San Jose, CA) and Image J v.1.45 was used to extract the areas stained by collagen deposits (15).
Next, the same analysis threshold was set for all groups $(0$, 5,10 , and 15 repetitions) to calculate type I collagen-stained areas (\%), as described (16). The collagen-stained area was quantified as a percentage of the total area of the tendon section (17). In addition, the cell numbers within collagen fibers were counted. The cellular density (cell count $/ \mathrm{mm}^{2}$ ) was quantified and the values were compared among the 4 groups. The mean value derived from the five different images was evaluated by two observers.

\section{Statistical Analysis}

All data were expressed as means \pm standard deviation (SD). Histological scoring was performed by 2 independent investigators, who were blinded to the results. Interclass correlation coefficients (ICCs) for intra-observer and inter-observer agreement were calculated. Differences among groups were compared using the Mann-Whitney U-test. Statistical significance was established at $\mathrm{P}<0.05$.

\section{RESULTS}

\section{Histological analysis}

Figure 2 shows the hematoxylin-eosin stained specimens for each number of scratches. As the number of scratches increased, cell counts decreased and fiber bundles sepa-
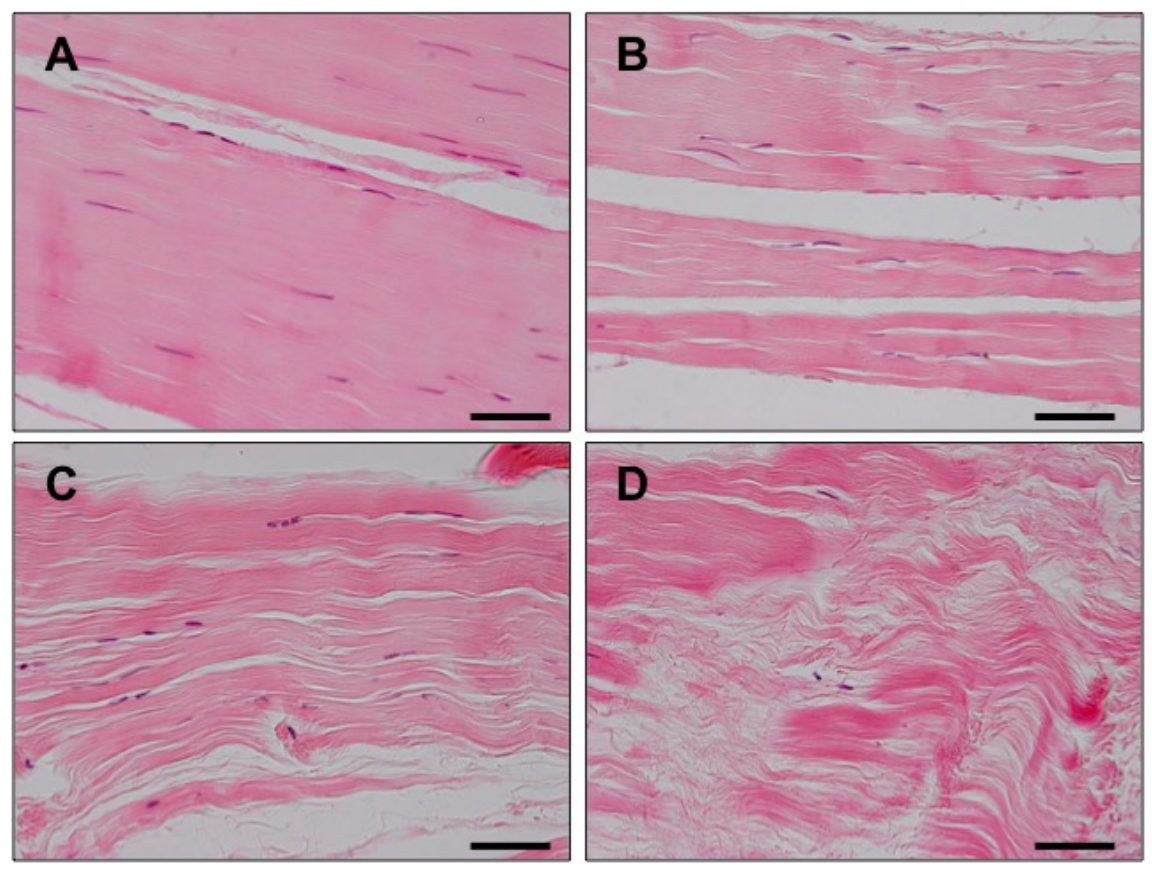

Figure 2. Histological photographs of the same semitendinosus (ST) tendons after hematoxylin-eosin staining (40x). (A) The control group (no scratching). (B) 5-repetition group. (C) 10-repetition group. (D) 15-repetition group. Bars, $50 \mu \mathrm{m}$. 
rated. As for vascularity, a small number of vascular structures were observed between fibers in the no scratching and 5 -repetition groups, with no vascular structures observed in the 10- and 15-repetition groups, the latter being scored as ' 0 ' on the Bonar scale. The modified Bonar score was $0.8 \pm 0.4$ for the control (no scratching group), increasing for the three scratching groups as follows: 5-repetitions, $3.2 \pm 1.5$; 10 -repetitions, $5.7 \pm 1.5$; and 15-repetitions, $7.2 \pm 0.4$ (Table I). The Bonar score increased as a function of the number of scratches (Figure 3A), with a significant difference in score between the control (no scratch) group and the 5 -repetition group $(\mathrm{P}=0.003)$, as well as between the 5 and 10-repetition groups $(\mathrm{P}=0.023)$. The Bonar score for collagen arrangement was significantly different between the three groups (Figure 3B), with cellularity being significantly increased between the 5- and 10-repetition groups $(\mathrm{P}=0.009)$. The intra-observer and inter-observer ICCs for the Bonar scoring system in this study were 0.82 and 0.77 , respectively.

\section{Immunohistochemical analysis}

The type I collagen-stained specimens are shown in Figure 4. The mean type I collagen-stained area was $88.4 \pm 5.8 \%$ in the control (no scratch) group, decreasing to $71.4 \pm 7.0 \%$ in the 5 -repetition group, $50.0 \pm 8.2 \%$ in the 10 -repetition group, and $35.6 \pm 6.8 \%$ in the 15 -repetition group (Table I). The type I collagen-stained area decreased as the number of scratches increased, with a significant difference between all groups $(\mathrm{P}<0.05$, Figure 5A). The mean cellular density was
$44.9 \pm 11.3 / \mathrm{mm}^{2}$ in the control (no scratch) group, decreasing to $38.7 \pm 11.6 / \mathrm{mm}^{2}$ in the 5 -repetition group, $17.6 \pm 5.7 /$ $\mathrm{mm}^{2}$ in the 10- repetition group, and $13.6 \pm 5.6 / \mathrm{mm}^{2}$ in the 15- repetition group (Table I). The density in the 10-repetition group was less than half the density in the 5-repetition group ( $\mathrm{p}=0.004$, Figure 5B). Intra- and inter-observer reliabilities were excellent (ICC $>0.91)$.

\section{DISCUSSION}

The most important finding in this study is that cleaning hamstring tendons by scratching causes histological alterations to the tendons. To our knowledge, this is the first histological analysis of artificial damage to the hamstring tendon during cleaning in the process of graft preparation for ACL reconstruction. In our tendon samples, the modified Bonar score increased as a function of the number of scratches performed, indicative of greater histological degeneration. Moreover, as the number of scratches increased, both the area of staining for type I collagen and the cellular density decreased.

Human biopsy studies have defined the stages of graft remodeling, recellularization, revascularization, and changes in collagen crimp and collagen fibril density during the proliferation phases, suggesting that the human ACL graft might have its lowest mechanical strength at 6-8 weeks after reconstruction (10). Three factors are known to exert adverse effects on the mechanical strength of the graft: increased revascularization and extracellular infiltration (18), loss of the regular collagen crimp pattern, and

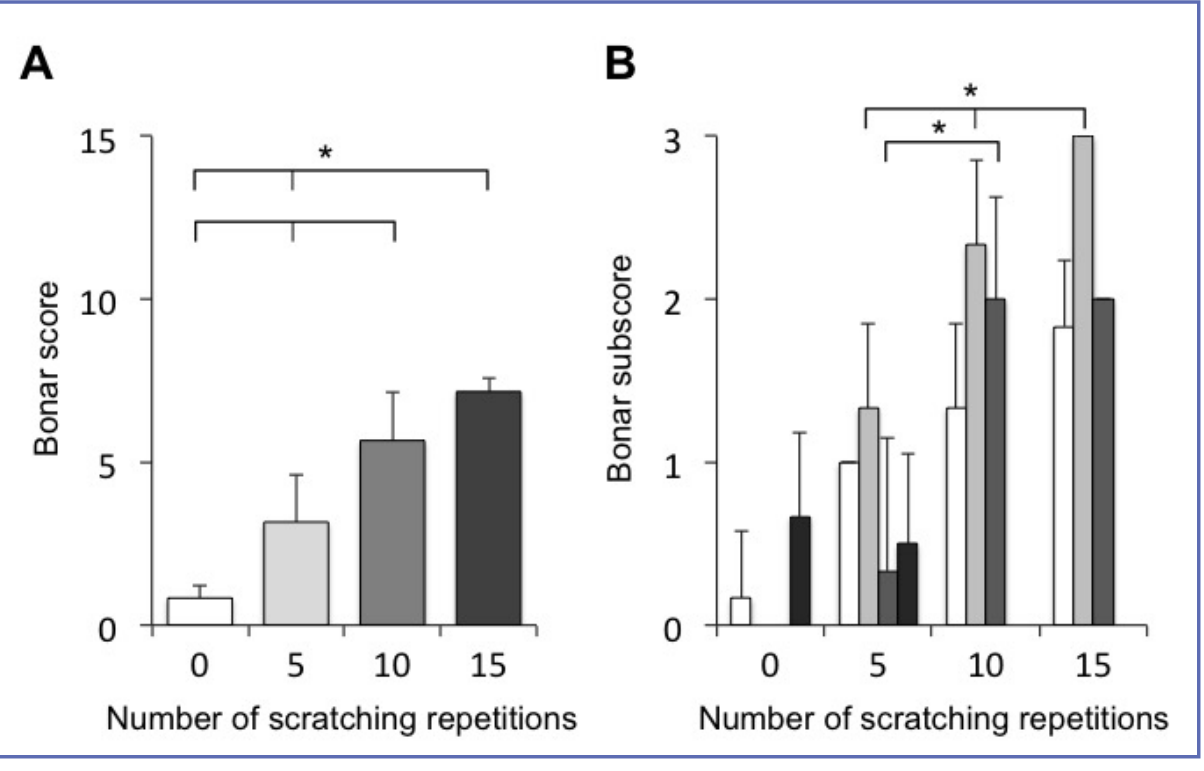

Figure 3. The Bonar score and the subscore analysis. (A) The Bonar score showed a progressive increase. (B) The subscore analysis presented, from left to right; changes in cell morphology (white), collagen alignment (light-gray), cellularity (dark-gray), and vascularity (black). 

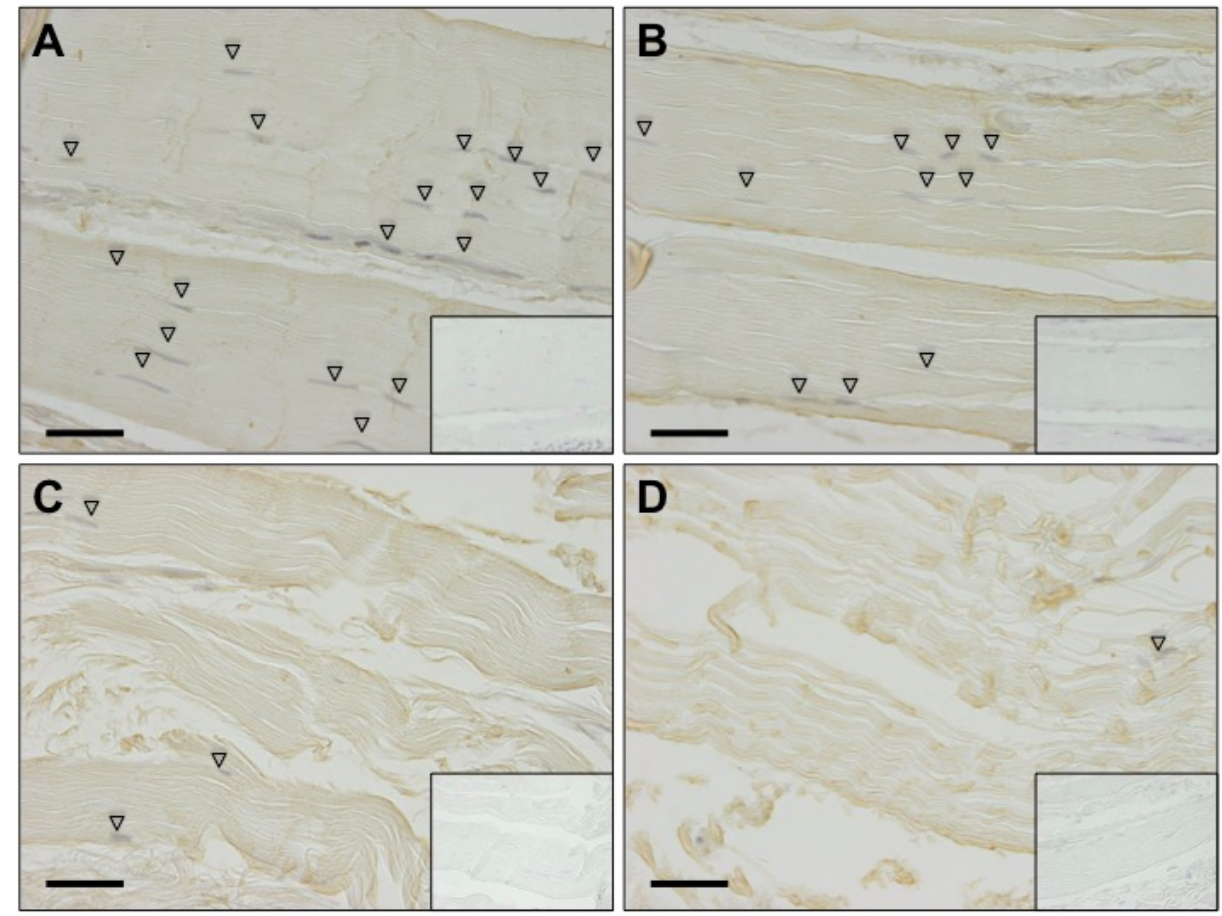

Figure 4. A tendon sample for type I collagen-staining. The triangles show spindle-shaped fibroblasts for the control (no scratching) group (A), and the 5- (B), 10- (C), and 15-repetition groups (D). Inlets, negative controls. Bars, $50 \mu \mathrm{m}$.

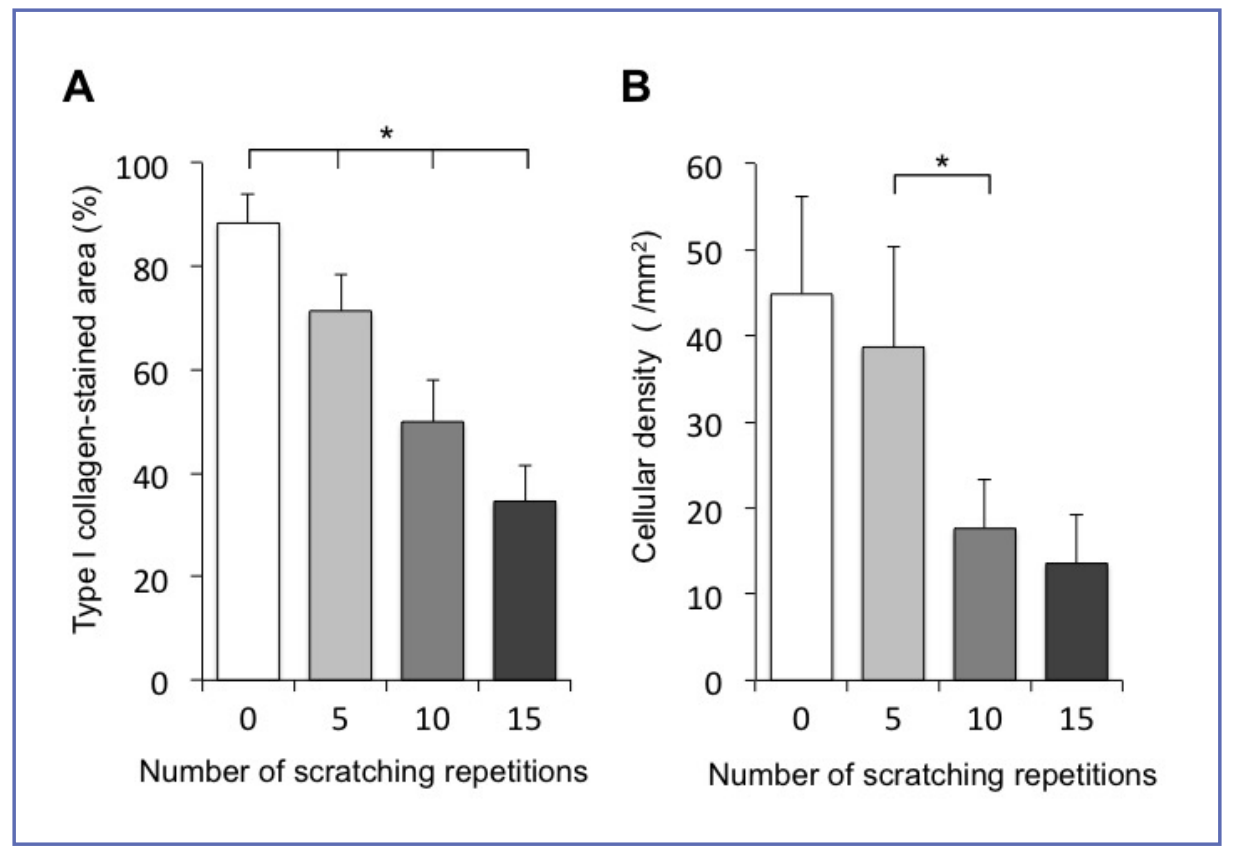

Figure 5. Immunohistochemical analysis. (A) Type I collagen-stained area (\%) decreased with significant differences between all the groups. (B) The cellular density was significantly lower for the 10- than 5 -repetition group; ${ }^{\star} \mathrm{P}<0.05$. 
Table I. Histological alterations for each scratching repetitions.

\begin{tabular}{|c|c|c|c|c|}
\hline \multirow[b]{2}{*}{ Variables } & \multicolumn{4}{|c|}{ Number of scratching repetitions } \\
\hline & 0 & 5 & 10 & 15 \\
\hline Bonar score (total) & $0.8 \pm 0.4$ & $3.2 \pm 1.5^{*}$ & $5.7 \pm 1.5^{*}$ & $7.2 \pm 0.4^{*}$ \\
\hline \multicolumn{5}{|l|}{ Bonar subscore } \\
\hline Cell morphology & $0.2 \pm 0.4$ & $1.0 \pm 0.1$ & $1.3 \pm 0.5$ & $1.8 \pm 0.4$ \\
\hline Collagen alignment & 0 & $1.3 \pm 0.5$ & $2.3 \pm 0.5^{*}$ & $3.0 \pm 0 *$ \\
\hline Cellularity & 0 & $0.3 \pm 0.8$ & $2.0 \pm 0.6^{*}$ & $2.0 \pm 0 *$ \\
\hline Vascularity & $0.7 \pm 0.5$ & $0.5 \pm 0.5$ & 0 & 0 \\
\hline Ground substance & 0 & 0 & 0 & 0 \\
\hline Type I collagen-stained area $(\%)$ & $88.4 \pm 5.8$ & $71.4 \pm 7.0^{*}$ & $50.0 \pm 8.2 *$ & $35.6 \pm 6.8^{*}$ \\
\hline Cellular density $(/ \mathrm{mm} 2)$ & $44.9 \pm 11.3$ & $38.7 \pm 11.6$ & $17.6 \pm 5.7^{*}$ & $13.6 \pm 5.6 *$ \\
\hline
\end{tabular}

decrease in collagen fibril density (19). Some authors have described that the decrease of collagen fibril density is associated with the maximum loss of graft strength and mechanical restitution of graft healing $(20,21)$. Moreover, a biopsy study has shown an increase in the collagen III content in the connective tissue of the hamstring autograft (22). Bosch et al. also found an increased expression of collagen type III in healing grafts (23). The mechanical strength of collagen III synthesis is lower than that of type I collagen (24). A previous review reported collagen III synthesis within the gaps between collagen fibrils, caused by damage to type I collagen fibrils (25). In this context, the decrease in the area of staining for type I collagen that we observed is indicative of a decrease in collagen fibril density during the proliferation phase. The greater damage to type I collagen by scratching would likely lead to more collagen III synthesis in collagen gaps, which could reduce the mechanical strength of the ACL hamstring autograft.

Fibroblasts have been shown to play an important role in the reconstruction of collagen fibrils (10). These cells are partially involved in collagen crimp formation and have been shown to have a significant impact on ACL graft remodeling (26). The cellular density of fibroblasts increases until the end of the proliferation phase, followed by a gradual decrease toward the density in the intact ACL $(19,20)$. It is also considered that the number of fibroblasts and their activity are an indicator of active ACL hamstring tendon autograft remodeling $(20,27)$. The mean cellular density in the present study was $38.9 / \mathrm{mm}^{2}$ in the 5 -repetition group and $17.6 / \mathrm{mm}^{2}$ in the 10-repetition group, causing a marked reduction of fibroblasts. This is also lower than the cellular density (30.2) of a graft measured 0.75 months after ACL reconstruction in a previous study (28). In addition, a systematic review found that the timeline of biological changes in grafts differs greatly between animals and humans. Graft remodeling in humans was found to be much slower compared to that in animals (29). Taken together, a marked decrease in the number of cells with $\geq 10$ scratches is indicative of the adverse effect of this extent of scratching on the final ligamentization phase of graft remodeling in humans.

This study has a number of limitations that need to be acknowledged. First is the small sample size, with a larger number of patients being needed to confirm our findings. Second, we did not measure the minimum amount of scratches necessary to remove all muscle tissues, which would have provided additional clinical implications for graft preparation. Third, we also did not perform histological assessment of the reconstruction implanted graft at the proliferation phase or the final phase of graft remodeling. It remains to be clarified as to whether the reduced tissue integrity in the pre-grafted tissue would always indicate deterioration or failure of tendon regeneration.

Based on our findings, we suggest that surgeons should limit scratching of the tendon with a curette to $<10$ repetitions because $\geq 10$ repetitions markedly reduced type I collagen-stained area and cellular density. Apart from the present study, no other basic research on the surgical tools used for tendon cleaning has been reported. Therefore, further study is required to compare the healing of the grafted tissues cleaned with a curette or with other tools such as scalpels. In conclusion, tendon cleaning by scratching during preparation of a hamstring autograft causes histological alternations to the tendon itself, with mainly a decrease in type I collagen fibrils and a reduction in the cellular density. We also conclude that excessive curettage, $\geq 10$ repetitions, could be deleterious to the tissue health of a hamstring autograft. 


\section{Conflict of interest}

The authors declare that they have no conflict of interest. (30)

\section{REFERENCES}

1. Lohmander LS, Englund PM, Dahl LL, Roos EM. The longterm consequence of anterior cruciate ligament and meniscus injuries: osteoarthritis. Am J Sports Med. 2007; 35:1756-1769.

2. Beynnon B, Johnson R, Abate J, Fleming B, Nichols C. Treatment of anterior cruciate ligament injuries, Part I. Am J Sports Med. 2005; 33:1579-1602.

3. Tandogan R, Taser O, Kayaalp A, Taşkiran E, et al. Analysis of meniscal and chondral lesions accompanying anterior cruciate ligament tears, relationship with age, time from injury, and level of sport. Knee Surg Sports Traumatol Arthrosc. 2004; 12:262-270.

4. Park SJ, Jung YB, Jung HJ, et al. Outcome of arthroscopic single- bundle versus double-bundle reconstruction of the anterior cruciate ligament: a preliminary 2-year prospective study. Arthroscopy. 2010; 26:630-636.

5. Tsuda E, Ishibashi Y, Fukuda A, Tsukada H, Toh S. Comparable results between lateralized single- and double-bundle ACL reconstructions. Clin Orthop Relat Res. 2009; 467:1042-1055.

6. Gorschewsky O, Klakow A, Riechert K, Pitzl M, Becker R. Clinical comparison of the Tutoplast allograft and autologous patellar tendon for the reconstruction of the anterior cruciate ligament: 2- and 6-year results. Am J Sports Med. 2005; 33:1202-1209.

7. Hamner DL, Brown CH Jr, Steiner ME, Hecker AT, Hayes WC. Hamstrings tendon grafts for reconstruction of the anterior cruciate ligament: biomechanical evaluation of the use of multiple strands and tensioning techniques. J Bone Joint Surg Am. 1999; 81:549-557.

8. Menetrey J, Duthon VB, Laumonier T, Fritschy D. "Biological failure" of the anterior cruciate ligament graft. Knee Surg Sports Traumatol Arthrosc. 2008; 16:224-231.

9. Amiel D, Kleiner JB, Roux RD, Harwood FL, Akeson WH. The phenomenon of "ligamentization": anterior cruciate ligament reconstruction with autogenous patellar tendon.

10. Scheffler SU, Unterhauser FN, Weiler A. Graft remodeling and ligamentization after cruciate ligament reconstruction. Knee Surg Sports Traumatol Arthrosc. 2008; 16:834-842.

11. Janssen RPA, Scheffler SU. Graft remodelling and ligamentization after anterior cruciate ligament reconstruction. In Prodromos CC The Anterior Cruciate Ligament: Reconstruction and Basic Science. 2nd Ed. Elsevier 2018:341-347.

12. Hippensteal KJ. Graft harvest techniques for knee ligament reconstruction. In: Johnson DL, ed. Master Techniques in Orthopaedic Surgery: Reconstructive Knee Surgery. Fourth edition. Philadelphia: Lippincott Williams and Wilkins, 2017:170-181.

13. Fearon A, Dahlstrom JE, Twin J, Cook J, Scott A. The Bonar score revisited: region of evaluation significantly influences the standardized assessment of tendon degeneration. J Sci Med Sport. 2014; 17:346-350.

\section{Acknowledgements}

We would like to thank Editage (www.editage.jp) for English language editing.

14. Inoue H, Furumatsu T, Maehara A, Tanaka T, Ozaki T. Histological and biological comparisons between complete and incomplete discoid lateral meniscus. Connect Tissue Res. 2016; 57:408-416.

15. Tang XN, Berman AE, Swanson RA, Yenari MA. Digitally quantifying cerebral hemorrhage using Photoshop and Image J. J Neurosci Methods. 2010; 190:240-243.

16. Varghese F, Bukhari AB, Malhotra R, De A. IHC Profiler: an open source plugin for the quantitative evaluation and automated scoring of immunohistochemistry images of human tissue samples. PLoS One. 2014; 9:e96801.

17. Park DY, Min BH, Choi BH, et al. The degeneration of meniscus roots is accompanied by bibrocartilage formation, which may precede meniscus root tears in osteoarthritic knees. Am J Sports Med. 2015; 43:3034-3044.

18. Tohyama H, Yasuda K. Extrinsic cell infiltration and revascularization accelerate mechanical deterioration of the patellar tendon after fibroblast necrosis. J Biomech Eng. 2001; 122:594-599.

19. Weiler A, Forster C, Hunt P, et al. The influence of locally applied platelet-derived growth factor-BB on free tendon graft remodeling after anterior cruciate ligament reconstruction. Am J Sports Med. 2004; 32:881-891.

20. Janssen RPA and Scheffler SU. Intra-articular remodelling of hamstring tendon grafts after anterior cruciate ligament reconstruction. Knee Surg Sports Traumatol Arthrosc. 2014; 22:2102-2108.

21. Weiler A, Peters G, Mäurer J, Unterhauser FN, Südkamp NP. Biomechanical properties and vascularity of an anterior cruciate ligament graft can be predicted by contrast-enhanced magnetic resonance imaging: a two-year study in sheep. Am J Sports Med. 2001; 29:751-761.

22. Petersen W, Laprell H. Insertion of autologous tendon grafts to the bone: a histological and immunohistochemical study of hamstring and patellar tendon grafts. Knee Surg Sports Traumatol Arthrosc. 2000;8:26-31.

23. Bosch U, Kasperczyk WJ, Oestern HJ, Tscherne H. The patellar tendon graft for PCL reconstruction. Morphological aspects in a sheep model. Acta Orthop Belg. 1994;60:57-61.

24. Frank C, Woo SL, Amiel D, Harwood F, Gomez M, Akeson W. Medial collateral ligament healing: A multidisciplinary assessment in rabbits. Am J Sports Med. 1983; 11:379-389.

25. Liu SH, Yang RS, al-Shaikh R, Lane JM. Collagen in tendon, ligament, and bone healing: A current review. Clin Orthop. 1995; (318):265-278.

26. Murray MM, Spector M. Fibroblast distribution in the anteromedial bundle of the human anterior cruciate ligament: the presence of alpha-smooth muscle actin-positive cells. J Orthop Res. 1999; 17:18-27.

27. Dustmann M, Schmidt T, Gangey I, Unterhauser FN, Weiler A, Scheffler SU. The extracellular remodeling of 
free-soft-tissue autografts and allografts for reconstruction of the anterior cruciate ligament: a comparison study in a sheep model. Knee Surg Sports Traumatol Arthrosc. 2008; 16:360-369.

28. Rougraff B, Shelbourne KD, Gerth PK, Warner J. Arthroscopic and histologic analysis of human patellar tendon autografts used for anterior cruciate ligament reconstruction. Am J Sports Med. 1993; 21:277-284.
29. Claes S, Verdonk P, Forsyth R, Bellemans J. The "ligamentization" process in anterior cruciate ligament reconstruction: what happens to the human graft? A systematic review of the literature. Am J Sports Med. 2011; 39: 2476-2483.

30. Padulo J., Oliva F., Frizziero A., Maffulli N. Muscles, Ligaments and Tendons Journal - Basic principles and recommendations in clinical and field Science Research: 2018 update. MLTJ 2018; 8(3): 305 - 307 